\title{
Effect of Inorganic N and P Fertilizers on Fruit Yield and Yield Components of Pineapple (Annanas comosus MERR L. Var. Smooth cayanne) at Jimma, Southwest Ethiopia
}

\author{
Tewodros M*, Mesfin S, Getachew W, Ashenafi A and Neim S
}

Jimma Agricultural Research Center, P.O. Box 192, Jimma, Ethiopia

\begin{abstract}
Field experiment was conducted at Jimma Agricultural Research Center (JARC) for two consecutive growing seasons (2012-2014 and 2015-2017 as a ratoon crop). The objective of the study was to evaluate the effect of different rates of inorganic $\mathrm{N}$ and $\mathrm{P}$ fertilizers on pineapple (variety Smooth cayenne) for yield and quality traits. Four levels of $\mathrm{N}\left(0,93.6,108\right.$ and $\left.281 \mathrm{~kg} \mathrm{ha}^{-1}\right)$ and four levels of $\mathrm{P}\left(0,134.8,269.6\right.$ and $404.4 \mathrm{~kg} \mathrm{P}_{2} \mathrm{O}_{5}$ ha-1 $\left.^{-1}\right)$ were arranged in RCBD with three replications. Data on yield and quality traits were collected and subjected to data analysis. Results of the study revealed that, the highest fruit yield was obtained by the rate of application of $281 \mathrm{~kg} \mathrm{~N} \mathrm{ha}^{-1}$ and $134.8 \mathrm{~kg} \mathrm{P}_{2} \mathrm{O}_{5} \mathrm{ha}^{-1}$. Application of nitrogen at a rate of $281 \mathrm{~kg} \mathrm{ha}^{-1}$ significantly increased fruit yield of pineapple up to $20.19 \%$ than the control. Similarly, phosphorus application at a rate of $134.8 \mathrm{~kg} \mathrm{P}_{2} \mathrm{O}_{5}$ ha $^{-1}$ increased significantly fruit yield by $68.22 \%$. For high yield and good quality of fruits, nitrogen should not be applied beyond $108 \mathrm{~kg} \mathrm{~N} \mathrm{ha}^{-1}$ rate. Apply treatments beyond $108 \mathrm{~kg} \mathrm{~N} \mathrm{ha}^{-1}$, the TSS content declined by $1.95 \%$. The economic analysis also revealed that the highest net benefit of $61,600.0$ Ethiopian Birr/ha (ETB/ha) with marginal rate of return of $237.0 \%$ was obtained by the application of $281 \mathrm{~kg} \mathrm{~N} \mathrm{ha}^{-1}$. Likewise, the net benefit of $12,320 \mathrm{ETB} / \mathrm{ha}$ with marginal rate of return of $507.0 \%$ were obtained by the application of $134.8 \mathrm{~kg} \mathrm{P}_{2} \mathrm{O}_{5} \mathrm{ha}^{-1}$. Based on the above results, a combined application of $281 \mathrm{~kg} \mathrm{~N}$ ha-1 $^{-1}$ and $134.8 \mathrm{~kg} \mathrm{P}_{2} \mathrm{O}_{5} \mathrm{ha}^{-1}$ are optimum and economically better for pineapple production at Jimma and its vicinity.
\end{abstract}

Keywords: Fertilizer rate; Nitrogen; Phosphorus; Pineapple; Quality

\section{Introduction}

Pineapple Annanas comosus (L.) is a perennial herb in the botanical family Bromeliaceae, native to the American tropics [1]. It is a hardy tropical fruit that grows well in frost-free area between $25^{\circ}$ north and south of the equator [2,3]. It is the second most important fruit crop after bananas and contributing over $20 \%$ of the world tropical fruits. The remaining $30 \%$ used as chunks, slices, juices, syrups, jams, crushed pineapple, diced pineapple in major producing countries [4-6]. Besides, wastes from processing of pineapple fruit are now further processed into sugar, wines, vinegar, animal feed during the dry season [7]. The leaves of pineapple have high quality fiber, manufacture of luxury clothing, making rope, fishing nets and pulp in the paper industry. In addition, the fruit of pineapple rich in digestible carbohydrates, fat, vitamins $\mathrm{A}, \mathrm{C}$ and essential minerals [8]. The suitability of pineapple as food stores on ships and medical ingredients greatly facilitated their distribution throughout the world. Currently, Annanas is a pan tropical genus and different species have been independently domesticated across continents.

In Ethiopia, pineapple successfully grows in South and Southwestern parts as small scale farming and the average yield of the crop is low about 45 tons/ha $[9,10]$ as compared to global average fruit yield of $67.5 \mathrm{t} / \mathrm{ha}$ [11]. This low yield is partly due to: low fertility status of the soil when the pineapple was grown, resulting from depletion by proceeding crops [12], lack of improved pineapple technologies for diverse environmental conditions, longer maturity, poor marketing system, presence of diseases and insect pests, and lack of improved post harvest handling technologies are a few to mention [13]. Besides, lack of sufficient information on the nutritional requirement of pineapple, leads low productivity [14]. According to FAO 2000 [11], pineapple requires nutrients such as nitrogen $(\mathrm{N})$, phosphorus $(\mathrm{P})$ and potassium (K) for fertility maintenance and crop production. These nutrients are specific in function and must be supplied in sufficient quantity to plant at the right time [7]. According to the reported of Spironello et al. [8], fruit yield of pineapple is very responsive to NPK fertilization. In fruit crops, it is known that applications of NPK fertilizer are vital for fruit yield and quality [3]. Besides, NPK plays an important role in the growth and development of the plant [11]. Despite the importance of NPK, the nutrient requirement of pineapple in Ethiopia is poorly understood and leads severe yield losses. To increase the productivity, better understanding on the importance of pineapple in association with its appropriate fertilizer requirements and types are needed to boost pineapple production, which minimize the poverty and improve the livelihood security of rural households. Therefore, this study was designed to determine the effect of inorganic nitrogen and phosphorus fertilization on the yield and quality traits of pineapple in Southwest Ethiopia.

\section{Materials and Methods}

\section{Description of the study area}

Field experiment was conducted at Jimma Agricultural Research Center (JARC) for two consecutive growing seasons (2012-2014 and

*Corresponding author: Tewodros Mulualem, Jimma Agricultural Research Center, P.O. Box 192, Jimma, Ethiopia, Tel: +251911748530; E-mail: tewodrosmulualem@gmail.com

Received May 22, 2018; Accepted June 20, 2018; Published June 27, 2018

Citation: Tewodros M, Mesfin S, Getachew W, Ashenafi A, Neim S (2018) Effect of Inorganic $\mathrm{N}$ and P Fertilizers on Fruit Yield and Yield Components of Pineapple (Annanas comosus MERR L. Var. Smooth cayanne) at Jimma, Southwest Ethiopia. Agrotechnology 7: 178. doi: 10.4172/2168-9881.1000178

Copyright: (C) 2018 Tewodros M, et al. This is an open-access article distributed under the terms of the Creative Commons Attribution License, which permits unrestricted use, distribution, and reproduction in any medium, provided the original author and source are credited. 
Citation: Tewodros M, Mesfin S, Getachew W, Ashenafi A, Neim S (2018) Effect of Inorganic N and P Fertilizers on Fruit Yield and Yield Components of Pineapple (Annanas comosus MERR L. Var. Smooth cayanne) at Jimma, Southwest Ethiopia. Agrotechnology 7: 178. doi: 10.4172/21689881.1000178

Page 2 of 6

2015-2017 as a ratoon crop). The center is located at latitude $7^{\circ} 40.00^{\prime}$ $\mathrm{N}$ and longitude $36^{\circ} 47^{\prime} .00^{\prime} \mathrm{E}$ with an altitude of 1753 m.a.s.l. The area receives mean annual rainfall of $1432 \mathrm{~mm}$ with maximum and minimum temperature of $26.5^{\circ} \mathrm{C}$ and $12.0^{\circ} \mathrm{C}$, respectively. The soil of the study area is characterized Eutric Nitosol (reddish brown) with $\mathrm{pH}$ of 5.3 [15].

\section{Soil sampling and analysis}

Fiften core soil samples randomly collected from $0-30 \mathrm{~cm}$ top soil and bulk to form a composite sample. The collected samples were air dried, crushed and allowed to pass through a $2 \mathrm{~mm}$ sieve. Particle size distribution was carried out by the hydrometer method, while soil $\mathrm{pH}$ in soil solution ratio $1: 2$ in $0.01 \mathrm{M} \mathrm{C}_{\mathrm{a}} \mathrm{Cl}_{2}$. Soil organic carbon was determined by the Walkey and Black method and total $\mathrm{N}$ by the micro-kjeldahl digestion method [16]. Available $\mathrm{P}$ was determined by Bremer and Mulraney, [16] extraction method. Exchabgeble bases were extracted with neutral $1 \mathrm{M} \mathrm{NH} \mathrm{OAC}_{4}$ at soil solution ratio of 1:10 and measured by flame photometry. Exchabgeble acidity was determined by titration of $1 \mathrm{M}$ KCL extract against $0.05 \mathrm{M} \mathrm{NaOH}$ to a pink end point using phenolphthalein as indicator [17] The soil sample analysis was conducted at JARC soil and plant tissue laboratory.

\section{Experimental materials, design and management}

For this study, the improved variety (Smooth cayenne) was planted in double row planting pattern of $90 \times 60 \times 30 \mathrm{~cm}$ between paired rows, between rows and plants, respectively during 2012-2014 and 20152017 as a ratoon crop. Treatment consisted on $\mathrm{N}$ applied at $(0,93.6$, 108 and $281 \mathrm{~kg} \mathrm{ha}^{-1}$ as urea $(46 \% \mathrm{~N})$ and $\mathrm{P}$ applied at $(0,134.8,269.6$ and $\left.404.4 \mathrm{~kg} \mathrm{ha}^{-1}\right)$ as DAP $\left(46 \% \mathrm{P}_{2} \mathrm{O}_{5}\right.$ and $\left.18 \% \mathrm{~N}\right)$.

The experiment was laid out in RCBD with three replications. The gross plot size for each treatment was $6 \mathrm{~m} \mathrm{x} 4 \mathrm{~m}\left(24 \mathrm{~m}^{2}\right)$. Plants was field established using double spaced of $30 \times 60 \times 90 \mathrm{~cm}$. Slips of the same size were used as planting material. Both fertilizers were applied near the rows. All DAP and $50 \%$ of urea was applied at planting. The remaining $50 \%$ of urea was applied as side banded after 60 days of planting. One month after planting, seedlings were earthed up, followed by frequent weeding. All other agronomic practices were followed according to the recommendations.

\section{Data collection and analysis}

Data were collected from ten plants from each plot and the average value was used for data analysis. The characters that manifested for data collection were: plant height $(\mathrm{m})$, leaf length $(\mathrm{cm})$, fruit length $(\mathrm{cm})$, fruit diameter $(\mathrm{cm})$, fruit fresh weight $\left(\mathrm{kg}\right.$ fruit $\left.{ }^{-1}\right)$ and TSS $(\%)$. Total soluble solid (TSS) in Brix was obtained by a temperature self compensating digital refractometer [18] at Jimma University, College of Agriculture and Veterinary Medicine (JUCAVM) food and nutrition laboratory. The collected data were subjected to analysis of variances and treatment means separated by Least Significant Difference (LSD) by using Statistical Analysis System (SAS) package [19].

\section{Economic evaluation}

Economic evaluation comprising partial budget with dominance and marginal rate of return was carried out. To estimate economic parameters, product (marketable fruit yield) was valued based on average market price collected from local markets during the two consecutive years of production when fresh pineapple fruits sold for 20 Ethiopian Birr per kg. Average price of urea, and DAP were 15.0 and 18.0 Birr per kg, respectively. Some of the concepts used in the partial budget analysis are gross field benefit (GFB), total variable cost (TVC) and the net benefit (NB). The GFB ha- ${ }^{-1}$ was obtained as the product of the real price and the mean pineapple fruit yield for each treatment. TVC refers to the sum of cost of all variable inputs (fertilizers) were used according to CIMMYT [20].

\section{Results and Discussion}

Data on the soil physico-chemical properties of Jimma was presented in Table 1. The data indicated, the soil of study area is sandy clay with low $\mathrm{N}$ and available $\mathrm{P}$, its characterization indicated soil pH 5.65 in water, $0.539 \mathrm{~g} \mathrm{~kg}^{-1} \mathrm{~N}, 3.27 \mathrm{~g} \mathrm{~kg}^{-1}$ organic C. $0.691 \mathrm{ppm}$ available $\mathrm{P}, 1.969$ meq/100 g K, $5.636 \%$ organic matter, $0.120 \mathrm{meq} / 100 \mathrm{~g}$ exchangeable acidity and $22.76 \mathrm{meq} / 100 \mathrm{~g}$ CEC. Particle size distributions were $52 \%$ sand, $36 \%$ clay and $12 \%$ silt.

The results of ANOVA indicated, plant height, leaf length, fruit length, fruit diameter, fruit fresh weight and TSS of pineapple plants had significant effect by the rates of nitrogen and phosphorus (Table 2). In both cropping seasons, plant height, fruit diameter, fruit fresh weight, and TSS of pineapple showed significant different at different level of fertilizer rates, while except fruit diameter, the rest of traits showed similar response in ratoon crop (Table 3). Application of 108 g Urea and $134.6 \mathrm{~g}$ DAP and $281.6 \mathrm{~g}$ Urea+134.8 g DAP produced the highest fruit yield of $1.35 \mathrm{~kg} /$ fruit and $1.31 \mathrm{~kg} /$ fruit from main and ratoon crop.

\section{The effect of NP fertilization on yield and quality traits of pineapple}

The effect of NP fertilizer on yield and quality traits of pineapple and ratoon crop at Jimma was presented in Table 4 and Table 5. The height of the plant is an important growth parameter directly linked with the fruit yield, which is positively correlated with plant productivity [21]. In this study, the effects of $\mathrm{N}$ fertilizer rates had significant effects on the height of pineapple plant (Tables 4 and 5). The tallest plant (58.81 $\mathrm{cm}$ ) was obtained from $108 \mathrm{~kg} \mathrm{~N} \mathrm{ha}^{-1}$ and $57.75 \mathrm{~cm}$ at rates of 269.6 $\mathrm{kg} \mathrm{P} \mathrm{ha}{ }^{-1}$. Pineapple treated at a control, the height became lowest $(51.48 \mathrm{~cm}$ and $53.32 \mathrm{~cm}) \mathrm{N}$ and $\mathrm{P}$ rates, respectively. The trends were $\mathrm{N}$ application at $108 \mathrm{~kg} \mathrm{~N} \mathrm{ha}^{-1}$ gave $10.29 \%$ and $1.5 \%$ increases over the control in plant and ratoon crops (Tables 4 and 5). This result is consistent with the report of [22] who reported, increasing the level of $\mathrm{N}$ application was observed to increase the height and the yield of pineapple plants. The application of $\mathrm{P}$ was showed similar trends like as $\mathrm{N}$ application on plant height. The tallest plants were obtained at 269.6 $\mathrm{kg} \mathrm{P} \mathrm{ha}{ }^{-1}$ rates. On contrary, the advanced rates of $\mathrm{N}$ and $\mathrm{P}$ from 93.6 $\mathrm{kg} \mathrm{N} \mathrm{ha}^{-1}$ to $108 \mathrm{~kg} \mathrm{~N} \mathrm{ha}^{-1}$ and $269.6 \mathrm{~kg} \mathrm{P} \mathrm{ha}^{-1}$ to $404.4 \mathrm{Kg} \mathrm{P} \mathrm{ha}^{-1}$ resulted

\begin{tabular}{|c|c|c|}
\hline No & Physico-chemical composition \\
\hline 1 & $\%$ Sand & 52 \\
\hline 2 & $\%$ Silt & 12 \\
\hline 3 & $\%$ Clay & 36 \\
\hline 4 & Textural class & Sandy clay \\
\hline 5 & $\mathrm{p}^{\mathrm{H}}\left(\mathrm{H}_{2} \mathrm{O}\right)(1: 2: 5)$ & 5.65 \\
\hline 6 & Organic carbon & 3.269 \\
\hline 7 & Available $\mathrm{P}(\mathrm{ppm})$ & 0.691 \\
\hline 8 & Total N $(\mathrm{g} / \mathrm{kg})$ & 0.539 \\
\hline 9 & Available $\mathrm{K}(\mathrm{meq} / 100 \mathrm{~g})$ & 1.969 \\
\hline 10 & $\%$ Organic matter & 5.636 \\
\hline 11 & Exchangeable acidity $(\mathrm{meq} / 100 \mathrm{~g})$ & 0.12 \\
\hline 12 & CEC $($ meq $/ 100 \mathrm{~g})$ & 22.76 \\
\hline 13 & Exchangeable $\mathrm{AL}+++(\mathrm{meq} / 100 \mathrm{~g})$ & Trace \\
\hline
\end{tabular}

Table 1: Physico-chemical properties of top soil $(0-30 \mathrm{~cm})$ of experimental fields of Jimma. 
Citation: Tewodros M, Mesfin S, Getachew W, Ashenafi A, Neim S (2018) Effect of Inorganic N and P Fertilizers on Fruit Yield and Yield Components of Pineapple (Annanas comosus MERR L. Var. Smooth cayanne) at Jimma, Southwest Ethiopia. Agrotechnology 7: 178. doi: 10.4172/21689881.1000178

Page 3 of 6

\begin{tabular}{|c|c|c|c|c|c|c|}
\hline Treatments & PH & LL & FL & FDi & FFW & Brix \\
\hline $0 \mathrm{~N}+0 \mathrm{P}_{2} \mathrm{O}_{5} \mathrm{~kg} / \mathrm{ha}$ & $57.0^{\mathrm{abcd}}$ & $58.21^{a}$ & $12.61^{\mathrm{a}}$ & $7.85^{\mathrm{ab}}$ & $1.19^{\mathrm{ab}}$ & $14.83^{\mathrm{ab}}$ \\
\hline $134.8 \mathrm{~kg} \mathrm{P} \mathrm{P}_{5} / \mathrm{ha}$ & $55.9^{\mathrm{bcd}}$ & $59.03^{a}$ & $11.63^{\mathrm{a}}$ & $8.00^{\mathrm{a}}$ & $1.06^{\mathrm{ab}}$ & $14.17^{b}$ \\
\hline $269.6 \mathrm{~kg} \mathrm{P} \mathrm{P}_{5} / \mathrm{ha}$ & $61.87^{a}$ & $57.01^{a}$ & $11.81^{\mathrm{a}}$ & $7.39^{\mathrm{bc}}$ & $1.09^{\mathrm{ab}}$ & $15.23^{a b}$ \\
\hline $404.4 \mathrm{~kg} \mathrm{P} \mathrm{P}_{2} \mathrm{O}_{5} / \mathrm{ha}$ & $53.86^{\mathrm{cd}}$ & $56.11^{a}$ & $12.03^{a}$ & $7.70^{\mathrm{abc}}$ & $0.99^{b}$ & $15.73^{a b}$ \\
\hline $93.6 \mathrm{~kg} \mathrm{~N} / \mathrm{ha}$ & $56.7^{\mathrm{abcd}}$ & $54.70^{\mathrm{a}}$ & $12.26^{a}$ & $7.18^{c}$ & $1.08^{\mathrm{ab}}$ & $14.43^{a b}$ \\
\hline $93.6 \mathrm{~kg} \mathrm{~N}+134.8 \mathrm{~kg} \mathrm{P} \mathrm{P}_{2} \mathrm{O}_{5} / \mathrm{ha}$ & $53.47^{\mathrm{cd}}$ & $55.78^{a}$ & $12.37^{a}$ & $7.28^{\mathrm{bc}}$ & $1.358^{\mathrm{a}}$ & $15.00^{\mathrm{ab}}$ \\
\hline $93.6 \mathrm{~kg} \mathrm{~N}+269.6 \mathrm{~kg} \mathrm{P} \mathrm{P}_{2} \mathrm{O}_{5} / \mathrm{ha}$ & $58.5^{\mathrm{abc}}$ & $56.67^{a}$ & $12.48^{a}$ & $7.65^{\mathrm{abc}}$ & $1.40^{\mathrm{a}}$ & $15.16^{a b}$ \\
\hline $93.6 \mathrm{~kg} \mathrm{~N}+404.4 \mathrm{~kg} \mathrm{P}_{2} \mathrm{O}_{5} / \mathrm{ha}$ & $55^{34 \mathrm{bcd}}$ & $58.59^{a}$ & $12.66^{\mathrm{a}}$ & $7.50^{\mathrm{abc}}$ & $1.09^{\mathrm{ab}}$ & 15.66 ab \\
\hline $108 \mathrm{~kg} \mathrm{~N} \mathrm{~kg} / \mathrm{ha}$ & $56.6^{\mathrm{abcd}}$ & $55.93^{a}$ & $12.36^{a}$ & $7.50^{a b c}$ & $1.15^{\mathrm{ab}}$ & $15.76 \mathrm{ab}$ \\
\hline 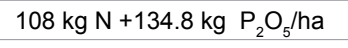 & $54.5^{\mathrm{bcd}}$ & $57.02^{\mathrm{a}}$ & $12.60^{a}$ & $7.51^{\mathrm{abc}}$ & $1.42^{\mathrm{a}}$ & $16.33^{a}$ \\
\hline $108 \mathrm{~kg} \mathrm{~N}+269.6 \mathrm{~kg} \mathrm{P}_{2} \mathrm{O}_{5} / \mathrm{ha}$ & $55.7^{\mathrm{bcd}}$ & $60.54^{a}$ & $12.64^{a}$ & $7.51^{\mathrm{abc}}$ & $1.22^{\mathrm{ab}}$ & $15.66^{a b}$ \\
\hline $108 \mathrm{~kg} \mathrm{~N}+404.4 \mathrm{~kg} \mathrm{P} \mathrm{P}_{5} / \mathrm{ha}$ & $56.5^{\mathrm{abcd}}$ & $58.25^{\mathrm{a}}$ & $12.13^{a}$ & $7.60^{\mathrm{abc}}$ & $1.11^{\mathrm{ab}}$ & $15.33^{a b}$ \\
\hline $281.6 \mathrm{~kg} \mathrm{~N} \mathrm{kg/ha}$ & $55.5^{\text {bcd }}$ & $57.54^{a}$ & $12.62^{a}$ & $7.64^{\mathrm{abc}}$ & $1.13^{\mathrm{ab}}$ & $15.83^{\mathrm{ab}}$ \\
\hline $281.6 \mathrm{~kg} \mathrm{~N}+134.8 \mathrm{~kg} \mathrm{P}_{2} \mathrm{O}_{5} / \mathrm{ha}$ & $57.3^{\mathrm{abcd}}$ & $55.94^{\mathrm{a}}$ & $12.33^{\mathrm{a}}$ & $7.61^{\mathrm{abc}}$ & $1.35^{\mathrm{a}}$ & $15.73^{a b}$ \\
\hline $281.6 \mathrm{~kg} \mathrm{~N}+269.6 \mathrm{~kg} \mathrm{P}_{2} \mathrm{O}_{5} / \mathrm{ha}$ & $59.82^{\mathrm{ab}}$ & $55.69^{a}$ & $12.40^{\mathrm{a}}$ & $7.58^{\mathrm{abc}}$ & $1.12^{\mathrm{ab}}$ & $15.566^{a b}$ \\
\hline $281.6 \mathrm{~kg} \mathrm{~N}+404.4 \mathrm{~kg} \mathrm{P}_{2} \mathrm{O}_{5} / \mathrm{ha}$ & $52.00^{\mathrm{d}}$ & $57.06^{a}$ & $12.29^{a}$ & $7.35^{\mathrm{bc}}$ & $1.15^{\mathrm{ab}}$ & $14.86^{a b}$ \\
\hline Mean & 56.28 & 57.13 & 12.33 & 7.55 & 1.18 & 15.33 \\
\hline $\begin{array}{c}\text { LSD }(<0.05) \\
\text { CV }(\%)\end{array}$ & $\begin{array}{l}3.51 \\
7.50\end{array}$ & $\begin{array}{l}2.91 \\
6.13\end{array}$ & $\begin{array}{l}0.87 \\
8.54\end{array}$ & $\begin{array}{l}0.38 \\
6.15\end{array}$ & $\begin{array}{c}0.18 \\
18.86\end{array}$ & $\begin{array}{l}0.85 \\
6.66\end{array}$ \\
\hline
\end{tabular}

PH: Plant height $(\mathrm{m})$; LL: Leaf length $(\mathrm{cm})$; FL: Fruit length $(\mathrm{cm})$; FDi: Fruit diameter $(\mathrm{cm})$; FW: Fruit fresh weight (kg/fruit) and Brix: TSS (\%)

Table 2: The analysis of variance of nitrogen and phosphorus on yield and yield related traits of pineapple 2012-2014 grown at Jimma.

\begin{tabular}{|c|c|c|c|c|c|c|}
\hline Treatments & PH & LL & FL & FDi & FFW & Brix \\
\hline $0 \mathrm{~N}+0 \mathrm{P}_{2} \mathrm{O}_{5} \mathrm{~kg} / \mathrm{ha}$ & $58.20^{\mathrm{cd}}$ & $53.42^{\mathrm{ab}}$ & $11.40^{\mathrm{b}}$ & $10.0^{\mathrm{a}}$ & $1.11^{\mathrm{ab}}$ & $15.16^{\mathrm{a}}$ \\
\hline $134.8 \mathrm{~kg} \mathrm{P}_{2} \mathrm{O}_{5} / \mathrm{ha}$ & $61.15^{\mathrm{abc}}$ & $54.57^{\mathrm{ab}}$ & $11.04^{\mathrm{ab}}$ & $10.0^{\mathrm{a}}$ & $0.88^{b}$ & $14.83^{\mathrm{a}}$ \\
\hline $269.6 \mathrm{~kg} \mathrm{P} \mathrm{P}_{5} / \mathrm{ha}$ & $60.3^{\text {abcd }}$ & $54.44^{\mathrm{ab}}$ & $11.68^{\mathrm{ab}}$ & $10.0^{\mathrm{a}}$ & $1.07^{\mathrm{ab}}$ & $15.16^{\mathrm{a}}$ \\
\hline $404.4 \mathrm{~kg} \mathrm{P} \mathrm{P}_{5} / \mathrm{ha}$ & $60.5^{\mathrm{abcd}}$ & $54.95^{\mathrm{ab}}$ & $12.01^{\mathrm{ab}}$ & $9.87^{a}$ & $0.85^{\mathrm{ab}}$ & $15.00^{\mathrm{a}}$ \\
\hline $93.6 \mathrm{~kg} \mathrm{~N} / \mathrm{ha}$ & $61.08^{\mathrm{abc}}$ & $57.69^{a}$ & $11.80^{\mathrm{ab}}$ & $9.14^{\mathrm{a}}$ & $0.95^{\mathrm{ab}}$ & $14.76^{\mathrm{a}}$ \\
\hline $93.6 \mathrm{~kg} \mathrm{~N}+134.8 \mathrm{~kg} \mathrm{P} \mathrm{P}_{2} \mathrm{O}_{5} / \mathrm{ha}$ & $62.84^{\mathrm{a}}$ & $56.28^{\mathrm{a}}$ & $11.86^{\mathrm{ab}}$ & $9.91^{\mathrm{a}}$ & $1.06^{\mathrm{ab}}$ & $15.16^{\mathrm{a}}$ \\
\hline $93.6 \mathrm{~kg} \mathrm{~N}+269.6 \mathrm{~kg} \mathrm{P} \mathrm{P}_{2} \mathrm{O}_{5} / \mathrm{ha}$ & $60.9^{\mathrm{abcd}}$ & $57.18^{a}$ & $12.28^{\mathrm{ab}}$ & $9.77^{\mathrm{a}}$ & $0.91^{b}$ & $15.16^{\mathrm{a}}$ \\
\hline $93.6 \mathrm{~kg} \mathrm{~N}+404.4 \mathrm{~kg} \mathrm{P}_{2} \mathrm{O}_{5} / \mathrm{ha}$ & $60.6^{\mathrm{abcd}}$ & $57.73^{a}$ & $11.66^{\mathrm{ab}}$ & $10.2^{\mathrm{a}}$ & $0.95^{\mathrm{ab}}$ & $15.50^{\mathrm{a}}$ \\
\hline $108 \mathrm{~kg} \mathrm{~N} \mathrm{~kg} / \mathrm{ha}$ & $61.98^{\mathrm{ab}}$ & $57.28^{a}$ & $11.80^{\mathrm{ab}}$ & $9.71^{\mathrm{a}}$ & $1.06^{\mathrm{ab}}$ & $15.00^{\mathrm{a}}$ \\
\hline $108 \mathrm{~kg} \mathrm{~N}+134.8 \mathrm{~kg} \mathrm{P} \mathrm{P}_{5} / \mathrm{ha}$ & $59.9^{\mathrm{abcd}}$ & $55.80^{\mathrm{ab}}$ & $12.11^{\mathrm{ab}}$ & $10.1^{\mathrm{a}}$ & $1.01^{\mathrm{ab}}$ & $15.33^{a}$ \\
\hline $108 \mathrm{~kg} \mathrm{~N}+269.6 \mathrm{~kg} \mathrm{P}_{2} \mathrm{O}_{5} / \mathrm{ha}$ & $60.4^{\mathrm{abcd}}$ & $50.63^{b}$ & $12.50^{\mathrm{a}}$ & $10.1^{\mathrm{a}}$ & $0.94^{\mathrm{ab}}$ & $15.83^{a}$ \\
\hline $108 \mathrm{~kg} \mathrm{~N}+404.4 \mathrm{~kg} \mathrm{P} \mathrm{P}_{2} \mathrm{O}_{5} / \mathrm{ha}$ & $61.17^{\mathrm{abc}}$ & $53.51^{\mathrm{ab}}$ & $11.92^{\mathrm{ab}}$ & $9.27^{\mathrm{a}}$ & $0.94^{\mathrm{ab}}$ & $15.16^{\mathrm{a}}$ \\
\hline $281.6 \mathrm{~kg} \mathrm{~N} \mathrm{~kg} / \mathrm{ha}$ & $59.93^{\text {bcd }}$ & $54.50^{\mathrm{ab}}$ & $11.50^{\mathrm{ab}}$ & $10.1^{\mathrm{a}}$ & $0.90^{\mathrm{b}}$ & $14.66^{a}$ \\
\hline 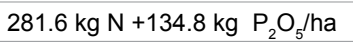 & $61.35^{\mathrm{abc}}$ & $53.85^{\mathrm{ab}}$ & $11.63^{\mathrm{ab}}$ & $9.98^{\mathrm{a}}$ & $1.31^{\mathrm{a}}$ & $15.16^{\mathrm{a}}$ \\
\hline $281.6 \mathrm{~kg} \mathrm{~N}+269.6 \mathrm{~kg} \mathrm{P}_{2} \mathrm{O}_{5} / \mathrm{ha}$ & $60.2^{\mathrm{abcd}}$ & $55.91^{\mathrm{ab}}$ & $11.95^{\mathrm{ab}}$ & $9.86^{a}$ & $0.88^{\mathrm{b}}$ & $15.16^{\mathrm{a}}$ \\
\hline $281.6 \mathrm{~kg} \mathrm{~N}+404.4 \mathrm{~kg} \mathrm{P} \mathrm{P}_{2} \mathrm{O}_{5} / \mathrm{ha}$ & $58.97^{\mathrm{cd}}$ & $55.89^{a b}$ & $11.81^{\mathrm{ab}}$ & $9.89^{a}$ & $0.90^{b}$ & $15.16^{\mathrm{a}}$ \\
\hline Mean & 60.60 & 55.23 & 11.81 & 9.87 & 0.98 & 15.15 \\
\hline $\begin{array}{c}\text { LSD }(<0.05) \\
\text { CV }(\%)\end{array}$ & $\begin{array}{l}1.43 \\
2.85\end{array}$ & $\begin{array}{l}2.71 \\
5.90\end{array}$ & $\begin{array}{l}0.67 \\
6.88\end{array}$ & $\begin{array}{l}0.62 \\
7.63\end{array}$ & $\begin{array}{c}0.19 \\
23.57\end{array}$ & $\begin{array}{l}0.60 \\
4.79\end{array}$ \\
\hline
\end{tabular}

PH: Plant height (m); LL: Leaf length (cm); FL: Fruit length (cm); FDi: Fruit diameter (cm); FW: Fruit fresh weight (kg/fruit) and Brix: TSS (\%)

Table 3: The analysis of variance of nitrogen and phosphorus on yield and yield related traits of ratoon pineapple grown at Jimma.

in a corresponding reduction in plant height, leaf length, fruit length and diameter, however, the opposite result was observed on fruit fresh weight and brix (TSS) contents, when the advanced rates of $\mathrm{N}$ and $\mathrm{P}$ [23]. Leaf length of a plant is an important growth character and directly linked with the yield potential of pineapple and positively correlated with plant productivity [7]. Pineapple tested with $281 \mathrm{~kg} \mathrm{~N} \mathrm{ha}^{-1}$ and 134 $\mathrm{kg} \mathrm{P} \mathrm{ha}{ }^{-1}$ had longest $(59.44 \mathrm{~cm}$ and $59.01 \mathrm{~cm})$ leaf length. The result obtained from this study was consistent with the report of [21] who reported, $58 \mathrm{~cm}$ and $52 \mathrm{~cm}$ of pineapple from application of $200 \mathrm{~kg} \mathrm{~N}$ $\mathrm{ha}^{-1}$ and $180 \mathrm{~kg} \mathrm{P} \mathrm{ha}^{-1}$. The longest leaf as a result of $\mathrm{N}$ application is indicative of the role of $\mathrm{N}$ in promoting vigorous foliage growth and more intense physiological activities in the plant which favored the synthesis of more assimilates for fruit development. Similarly, nitrogen increases the chlorophyll contents of the leaves, thereby promote the photosynthetic capacity of the plant, plays a part in the manufacture of proteins and responsible for high fruit yield in pineapple [4]. On the other hand, phosphorous promotes $\mathrm{CO}_{2}$ assimilation and energy for the translocation of carbohydrates from leaves to the fruits of crops, where carbohydrates are the main storage material [24].

Likewise, fruit length, fruit diameter and fresh fruit yield followed similar trends as plant height. The control plots $(0 \mathrm{Kg} \mathrm{N}$ and $0 \mathrm{Kg}$ P) showed the shortest plants with the lowest fruit length and fruit diameter. Fruit length and diameter had highest at application of 108 $\mathrm{kg} \mathrm{N} / \mathrm{ha}$ rates were statistically similar (Table 4). Fruit length and diameter/fruit were significantly increased by $\mathrm{P}$ application up to the $269.6 \mathrm{~kg} \mathrm{ha}^{-1}$ rate and not beyond. Typically, the rate of application of $\mathrm{N}$ 
Citation: Tewodros M, Mesfin S, Getachew W, Ashenafi A, Neim S (2018) Effect of Inorganic N and P Fertilizers on Fruit Yield and Yield Components of Pineapple (Annanas comosus MERR L. Var. Smooth cayanne) at Jimma, Southwest Ethiopia. Agrotechnology 7: 178. doi: 10.4172/21689881.1000178

Page 4 of 6

\begin{tabular}{|c|c|c|c|c|c|c|}
\hline \multirow{2}{*}{ Treatments } & \multicolumn{6}{|c|}{$2012 / 2014$} \\
\hline & PH & LL & FL & FDi & FFW & Brix \\
\hline \multicolumn{7}{|l|}{ Nitrogen (Kg/ha) } \\
\hline 0 & $51.48^{\mathrm{b}}$ & $54.54^{\mathrm{b}}$ & $12.24^{\mathrm{ab}}$ & $7.24^{b}$ & $1.04^{b}$ & $14.83^{\mathrm{ab}}$ \\
\hline 93.6 & $54.45^{\mathrm{ab}}$ & $56.90^{\mathrm{ab}}$ & $12.32^{\mathrm{ab}}$ & $7.45^{\mathrm{ab}}$ & $1.07^{\mathrm{ab}}$ & $14.43^{\mathrm{ab}}$ \\
\hline 108 & $56.30^{\mathrm{ab}}$ & $56.63^{\mathrm{ab}}$ & $12.44^{\mathrm{a}}$ & $7.61^{\mathrm{a}}$ & $1.18^{\mathrm{a}}$ & $15.49^{a}$ \\
\hline 281 & $58.81^{a}$ & $59.44^{a}$ & $12.31^{\mathrm{ab}}$ & $7.42^{\mathrm{ab}}$ & $1.25^{\mathrm{a}}$ & $15.83^{\mathrm{a}}$ \\
\hline SE \pm & 4.22 & 3.50 & 1.05 & 0.46 & 0.22 & 1.02 \\
\hline \multicolumn{7}{|c|}{ Phosphorous ( $\mathrm{P}_{\underline{2}} \underline{\mathrm{O}}_{5} \underline{\mathrm{Kg} / \mathrm{ha})}$} \\
\hline 0 & $53.32^{\mathrm{b}}$ & $56.01^{\mathrm{b}}$ & $11.78^{b}$ & $7.39^{\mathrm{ab}}$ & $1.07^{b}$ & $14.83^{\mathrm{ab}}$ \\
\hline 134.8 & $55.19^{\mathrm{ab}}$ & $59.01^{a}$ & $12.62^{\mathrm{a}}$ & $7.58^{\mathrm{a}}$ & $1.19^{a b}$ & $14.17^{b}$ \\
\hline 269.6 & $57.75^{\mathrm{a}}$ & $57.37^{\mathrm{ab}}$ & $12.49^{a}$ & $7.56^{a}$ & $1.28^{\mathrm{a}}$ & $15.23^{\mathrm{ab}}$ \\
\hline 404.4 & $57.16^{a}$ & $56.16^{\mathrm{ab}}$ & $12.28^{\mathrm{ab}}$ & $7.58^{\mathrm{a}}$ & $1.19^{\mathrm{ab}}$ & $15.73^{\mathrm{ab}}$ \\
\hline Interaction $\mathrm{N} \times \mathrm{P}$ & NS & NS & NS & NS & NS & NS \\
\hline CV (\%) & 7.50 & 6.13 & 8.54 & 6.15 & 18.86 & 6.66 \\
\hline
\end{tabular}

$\mathrm{PH}$ : Plant height $(\mathrm{m})$; LL: Leaf length $(\mathrm{cm})$; FL: Fruit length $(\mathrm{cm})$; FDi: Fruit diameter $(\mathrm{cm})$; FW: Fruit fresh weight (kg/fruit) and Brix: TSS (\%).

Table 4: Effect of nitrogen and phosphorus on yield and yield related traits of pineapple crop grown at Jimma.

\begin{tabular}{|c|c|c|c|c|c|c|}
\hline \multirow{2}{*}{ Treatments } & \multicolumn{6}{|c|}{ Ratoon crop } \\
\hline & PH & LL & FL & FDi & FFW & Brix \\
\hline \multicolumn{7}{|l|}{ Nitrogen (Kg/ha) } \\
\hline 0 & $60.02^{b}$ & $54.34^{\mathrm{ab}}$ & $11.43^{b}$ & $9.65^{\mathrm{ab}}$ & $0.87^{\mathrm{ab}}$ & $15.14^{\mathrm{ab}}$ \\
\hline 93.6 & $60.12^{\mathrm{b}}$ & $58.22^{\mathrm{a}}$ & $12.08^{\mathrm{a}}$ & $9.98^{a}$ & $0.93^{\mathrm{ab}}$ & $14.76^{\mathrm{ab}}$ \\
\hline 108 & $61.35^{\mathrm{ab}}$ & $54.30^{\mathrm{ab}}$ & $11.90^{\mathrm{ab}}$ & $9.75^{\mathrm{ab}}$ & $1.09^{a}$ & $15.00^{\mathrm{a}}$ \\
\hline 281 & $60.89^{a}$ & $55.03^{\mathrm{ab}}$ & $11.72^{\mathrm{ab}}$ & $9.95^{\mathrm{a}}$ & $1.00^{\mathrm{a}}$ & $14.66^{\mathrm{ab}}$ \\
\hline SE \pm & 1.72 & 3.26 & 0.81 & 0.75 & 0.23 & 0.72 \\
\hline \multicolumn{7}{|c|}{ Phosphorous ( $\mathbb{P}_{2} \underline{\mathrm{O}}_{5} \underline{\mathrm{Kg} / \mathrm{ha})}$} \\
\hline 0 & $60.03^{b}$ & $53.72^{\mathrm{ab}}$ & $11.62^{\mathrm{ab}}$ & $9.43^{b}$ & $0.91^{\mathrm{ab}}$ & $14.81^{\mathrm{b}}$ \\
\hline 134.8 & $61.33^{a}$ & $57.13^{a}$ & $11.66^{\mathrm{ab}}$ & $10.00^{\mathrm{a}}$ & $0.95^{\mathrm{ab}}$ & $14.83^{a}$ \\
\hline 269.6 & $60.46^{a b}$ & $54.54^{\mathrm{ab}}$ & $12.19^{\mathrm{a}}$ & $9.95^{\mathrm{a}}$ & $1.03^{a}$ & $15.16^{\mathrm{a}}$ \\
\hline 404.4 & $60.30^{\mathrm{ab}}$ & $55.53^{\mathrm{ab}}$ & $11.85^{\mathrm{ab}}$ & $9.78^{\mathrm{ab}}$ & $1.16^{\mathrm{a}}$ & $15.00^{\mathrm{ab}}$ \\
\hline Interaction $\mathrm{N} \times \mathrm{P}$ & NS & NS & NS & NS & NS & NS \\
\hline CV\% & 2.85 & 5.90 & 6.88 & 7.63 & 23.57 & 4.79 \\
\hline
\end{tabular}

$\mathrm{PH}$ : Plant height $(\mathrm{m})$; LL: Leaf length $(\mathrm{cm})$; FL: Fruit length $(\mathrm{cm}) ; \mathrm{FDi}$ : Fruit diameter $(\mathrm{cm})$; FW: Fruit fresh weight (kg/fruit) and Brix: TSS (\%)

Table 5: Effect of nitrogen and phosphorus on yield and yield related traits of ratoon pineapple grown at Jimma.

from 0 up to $108 \mathrm{~kg} \mathrm{ha}^{-1}$, increased the length of fruit by $1.63 \%$, whereas, the application of $\mathrm{P}$ up to $269.6 \mathrm{Kg} \mathrm{ha}^{-1}$, gave the corresponding values of $6.02 \%$ than the control. Fruit length and diameter followed similar trends as increased the NP rates from $0 \mathrm{~kg} \mathrm{~N}^{-1}$ to $108 \mathrm{~kg} \mathrm{~N}^{-1}$ and $0 \mathrm{~kg} \mathrm{P} \mathrm{ha-1}$ to $269.6 \mathrm{~kg} \mathrm{P} \mathrm{ha}^{-1}$, respectively. The superior growth attributes was obtained at high rates of $\mathrm{N}$ and $\mathrm{P}$ in this study has been reported by other researchers $[4,21]$. The positive response of growth characters to the applied plant nutrients is attributable to their role in cell division, multiplication and photosynthesis which gave rise to increase in size and length of leaves, fruit and stems. Furthermore, the positive response shown by yield parameters to $\mathrm{N}$ and $\mathrm{P}$ could be directly linked to the well-developed photosynthetic surfaces and increased physiological activities leading to more assimilates being produced and subsequently translocation of assimilates and utilized for fast fruit development. In this regard, [25] reported, both $\mathrm{N}$ and $\mathrm{P}$ are necessary for root initiation, elongation, and increase in fruit length, diameter and fruit yield. Total fruit fresh weight obtained at $281 \mathrm{~kg} \mathrm{~N}$ $\mathrm{ha}^{-1}$ rates showed an increase of $20.19 \%$ over that of $0 \mathrm{~kg} \mathrm{~N} \mathrm{ha}^{-1}$ rate, whereas, increasing $\mathrm{P}$ rates from 0 to $269.6 \mathrm{~kg} \mathrm{ha}^{-1}$, increase fresh fruit weight by $68.22 \%$; a further increase P application up to $404.4 \mathrm{~kg} \mathrm{ha}^{-1}$ decreased fruit fresh weight by 11.21 percent. On contrary, the results obtained from this study is higher than the reported value of $127.04 \mathrm{~kg}$
$\mathrm{N} \mathrm{ha}^{-1}$ and $65 \mathrm{~kg} \mathrm{P} \mathrm{ha}^{-1}$ with fruit fresh weight of $16.72 \mathrm{t} \mathrm{ha}^{-1}$ [26]. The observed disparity between the results could be explained on the basis of the soil fertility difference and the environmental conditions upon which the plant was grown. Besides, [23], also reported significant differences on fruit yield of pineapple due to N, P and K application. This result was consistent with the report of and [27] who suggested that the maintenance dressing of $270 \mathrm{~kg} \mathrm{~N} \mathrm{ha}^{-1}$ and $280 \mathrm{~kg} \mathrm{P} \mathrm{ha}^{-1}$ per cropping season may be adequate for continuous pineapple production in Nigeria.

The effect of $\mathrm{N}$ fertilizer rates on fruit juice quality of pineapple are presented in Tables 4 and 5. The TSS content increased significantly as $\mathrm{N}$ rates increased at ratoon, but no significant effect in the main plant. According to $[7,28,29]$ reported, high $\mathrm{N}$ fertilizer application had significant positive effect on the TSS content. In this study, the highest TSS content (15.49\%) and (15.33\%) was obtained at $\mathrm{N}$ rates of $108 \mathrm{~kg} \mathrm{~N} \mathrm{ha}^{-1}$ in plant and ratoon crops, respectively. Apply treatments beyond $108 \mathrm{~kg} \mathrm{~N} \mathrm{ha}^{-1}$, the TSS content declined by $1.95 \%$. This result was consistent with the report of Ademar et al. [23] who reported, high $\mathrm{N}$ application had positive effect on fruit yield, but decreased quality (TSS) contents significantly. On contrary, adequate supply of $\mathrm{P}$ is important for energy synthesis and translocation, and it also increases yield and improves the fruit quality (brix contents). Hence, the positive 
Citation: Tewodros M, Mesfin S, Getachew W, Ashenafi A, Neim S (2018) Effect of Inorganic N and P Fertilizers on Fruit Yield and Yield Components of Pineapple (Annanas comosus MERR L. Var. Smooth cayanne) at Jimma, Southwest Ethiopia. Agrotechnology 7: 178. doi: 10.4172/21689881.1000178

Page 5 of 6

\begin{tabular}{|c|c|c|c|c|c|c|c|c|c|c|}
\hline \multicolumn{11}{|c|}{ Sensitivity Analysis } \\
\hline $\begin{array}{l}\text { Fertilizer } \\
\text { level }\end{array}$ & $\begin{array}{l}\text { Mean Yield } \\
\text { (Kg/ha) }\end{array}$ & \begin{tabular}{|c|} 
Adjusted Yield \\
(Kg/ha)
\end{tabular} & $\begin{array}{c}\text { Gross Benefit } \\
\text { (ETB/ha) }\end{array}$ & $\begin{array}{c}\text { Cost of Urea } \\
\text { (ETB/ha) }\end{array}$ & $\begin{array}{l}\text { Cost Of DAP } \\
\text { (ETB/ha) }\end{array}$ & $\begin{array}{c}\text { Gross Cost } \\
\text { (ETB/ha) }\end{array}$ & $\begin{array}{l}\text { Net Benefit } \\
\text { (ETB/ha) }\end{array}$ & $\begin{array}{c}\text { Change } \\
\text { gross cost }\end{array}$ & $\begin{array}{c}\text { Change } \\
\text { Net benefit }\end{array}$ & $\begin{array}{r}\text { MRR } \\
(\%)\end{array}$ \\
\hline \multicolumn{11}{|l|}{ N(kg/ha) } \\
\hline 0 & 50160.0 & 5016.0 & 100320.0 & 0 & 0 & 0 & 100320.0 & - & - & - \\
\hline 93.6 & 51480.0 & 5148.0 & 102960.0 & 1404.0 & 0 & 1404.0 & 102960.0 & 1404.0 & 2640.0 & 188.0 \\
\hline 108 & 51920.0 & 5192.0 & 103840.0 & 1620.0 & 0 & 1620.0 & 103840.0 & 216.0 & 880.0 & 407.0 \\
\hline 281 & 55000.0 & 5500.0 & 110000.0 & 4215.0 & 0 & 4215.0 & 110000.0 & 2595.0 & 61600.0 & 237.0 \\
\hline \multicolumn{11}{|c|}{$\mathrm{P}\left(\mathrm{P}_{2} \mathrm{O}_{5} \mathrm{~kg} / \mathrm{ha}\right)$} \\
\hline 0 & 35640.0 & 3564.0 & 71280.0 & 0 & 0 & 0 & 71280.0 & - & - & - \\
\hline 134.8 & 41800.0 & 4180.0 & 83600.0 & 0 & 2426.0 & 2426.0 & 83600.0 & 2426.0 & 12320.0 & 507.0 \\
\hline 269.6 & 42680.0 & 4268.0 & 85360.0 & 0 & 4852.8 & 4852.8 & 85360.0 & 2426.0 & 1760.0 & 72.0 \\
\hline 404.4 & 48400.0 & 4840.0 & 96800.0 & 0 & 7279.2 & 7279.2 & 96800.0 & 2426.0 & 11400.0 & 470.0 \\
\hline $\begin{array}{l}\text { Fertilizer } \\
\text { level }\end{array}$ & $\begin{array}{l}\text { Mean Yield } \\
(\mathrm{Kg} / \mathrm{ha})\end{array}$ & $\begin{array}{c}\text { Adjusted Yield } \\
(\mathrm{Kg} / \mathrm{ha})\end{array}$ & $\begin{array}{c}\text { Gross Benefit } \\
\text { (ETB/ha) }\end{array}$ & $\begin{array}{c}\text { Cost of Urea } \\
\text { (ETB/ha) }\end{array}$ & $\begin{array}{l}\text { Cost Of DAP } \\
\text { (ETB/ha) }\end{array}$ & $\begin{array}{c}\text { Gross Cost } \\
(+10 \%)\end{array}$ & $\begin{array}{l}\text { Net Benefit } \\
(-10 \%)\end{array}$ & $\begin{array}{c}\text { Change } \\
\text { gross cost }\end{array}$ & $\begin{array}{c}\text { Change } \\
\text { Net benefit }\end{array}$ & $\begin{array}{r}\text { MRR } \\
(\%)\end{array}$ \\
\hline \multicolumn{11}{|l|}{ N(Kg/ha) } \\
\hline 0 & 50160.0 & 5016.0 & 100320.0 & 0 & 0 & - & 90288.0 & - & - & - \\
\hline 93.6 & 51480.0 & 5148.0 & 102960.0 & 1404.0 & 0 & 1544.00 & 92664.0 & 1544.0 & 2376.0 & 153.86 \\
\hline 108 & 51920.0 & 5192.0 & 103840.0 & 1620.0 & 0 & 1782.00 & 93456.0 & 238.00 & 792.0 & 332.77 \\
\hline 281 & 55000.0 & 5500.0 & 110000.0 & 4215.0 & 0 & 4636.00 & 99000.0 & 2854.0 & 5544.0 & 194.25 \\
\hline \multicolumn{11}{|c|}{$\mathrm{P}\left(\mathrm{P}_{2} \mathrm{O}_{5} \mathrm{Kg} / \mathrm{ha}\right)$} \\
\hline 0 & 35640.0 & 3564.0 & 71280.0 & 0 & 0 & 0 & 64152.0 & - & - & - \\
\hline 134.8 & 41800.0 & 4180.0 & 83600.0 & 0 & 2426.0 & 2668.0 & 75240.0 & 2668.0 & 11088.0 & 415.59 \\
\hline 269.6 & 42680.0 & 4268.0 & 85360.0 & 0 & 4852.8 & 5338.0 & 76824.0 & 2670.0 & 1584.0 & 59.32 \\
\hline 404.4 & 48400.0 & 4840.0 & 96800.0 & 0 & 7279.2 & 8006.2 & 87120.0 & 2668.2 & 10296.0 & 285.90 \\
\hline
\end{tabular}

-MRR: Marginal Rate of Return; field price of pineapple: $20 \mathrm{ETB}^{-1} \mathrm{ha}^{-1}$; price of urea $=15 \mathrm{ETB} \mathrm{ha}^{-1}$; price of DAP: $18 \mathrm{ETB} / \mathrm{kg}$.

Table 6: Marginal rate of return and sensitivity analysis for NP fertilizer at Jimma stations.

response of fruit yield and yield components to increased rates of $\mathrm{N}$ and $P$ could be adduced to high energy synthesis and translocation activities stimulated by $\mathrm{N}$ and $\mathrm{P}$ application. Moreover, the experimental soils were slightly low in nitrogen content, hence, the positive response observed. However, the $\mathrm{pH}$ of the experimental sites was slightly acidic (5.65), and had some fixation of $\mathrm{P}$ in the soil solution, as a result, some difficulty to absorb and utilize available nitrogen and other essential mineral nutrients from the soil by plants.

The results of economic analysis revealed that, the highest change net benefit of $61,600.0 \mathrm{ETB} /$ ha with marginal rate of return (MMR) of $237.0 \%$ and $12,320.0 \mathrm{ETB} \mathrm{ha}^{-1}$ with marginal rate of return of $507.0 \%$ were obtained by application of $281 \mathrm{~kg} \mathrm{~N}$ and $134.8 \mathrm{~kg} \mathrm{P}_{2} \mathrm{O}_{5} \mathrm{ha}^{-1}$, respectively. An increase in output will always raise profit as long as the marginal rate of return is higher than the minimum rate of return i.e. 50 to $100 \%$. Data in Table 6 showed that, the marginal rate of return at the nitrogen application rate of $281 \mathrm{~kg} \mathrm{~N} \mathrm{ha}^{-1}$ was greater than $50 \%$ marginal rate of return showed an economically feasible. The MRR decreased as the cost increased. Besides, the marginal rate of return due to phosphorus application is also more than $50 \%$, application of phosphorus fertilizer is economically profitable up to the rate of 134.8 $\mathrm{kg} \mathrm{P}_{2} \mathrm{O}_{5} \mathrm{ha}^{-1}$. Sensitivity analysis of profitability of fertilizer use relative to $10 \%$ increase in fertilizer price remained feasible (Table 6). Similarly, it remained profitable up on $10 \%$ yield decrease due to moisture loss during transportation and storage. In both cases MRR were above the acceptable range. This depicts relative advantage and stability of economic benefits due to NP fertilizer use in the production of pineapple in Jimma and other areas of similar soil and climatic conditions.

\section{Conclusion and Recommendation}

The present findings showed that nitrogen and phosphorus had positive effects on growth, yield and quality of pineapple. The application of $281 \mathrm{~kg} \mathrm{~N} h a^{-1}$ and $134.8 \mathrm{~kg} \mathrm{P}_{2} \mathrm{O}_{5} \mathrm{ha}^{-1}$ had significantly increased the fruit yield of pineapple. The economic analysis reveals that further application of NP fertilizer is not economical. Thus, application of $281 \mathrm{~kg} \mathrm{~N} \mathrm{ha}^{-1}$ and $134.8 \mathrm{~kg} \mathrm{P}_{2} \mathrm{O}_{5} \mathrm{~kg} \mathrm{ha}^{-1}$ is economical and recommended for pineapple production under Jimma and its vicinity of Southwest Ethiopia.

\section{Acknowledgement}

The authors would like to acknowledge Ethiopian Institute of Agricultural Research (EIAR)/ Jimma Agricultural Research Center (JARC), for the financial support of this study.

\section{References}

1. Bartholomew DP, Rohrbach KG, Evans DO (2002) Pineapple cultivation in Hawaii. College of Tropical Agriculture and Human Resources, University of Hawaii. Fruits and Nuts.

2. Samson JA (1986) Tropical Fruits ( $2^{\text {nd }}$ edn) Longman Scientific and Technical Tropical Agriculture Series. pp: 190-215.

3. Vho S (1999) Pineapple production practices. National Agricultural Research Institute, Mon Repos, East Coast Demerara.

4. Mohamed $\mathrm{MH}$, Ahmad $\mathrm{H}$ (2004) Nutrient supply and dry-matter partitioning of pineapple cv. Josapine on sandy tin tailings. Technical paper, Department of Land Management, Faculty of Agriculture, University of Putra, Malaysia.

5. FAO (2008) Fertilizer and their use. FAN Rome, Italy.

6. Omotoso OS, Kinrinde EA (2013) Effect of nitrogen fertilizer on some growth, yield and fruit quality parameters in pineapple (Annanas comosus (L.) Merr.) Plant at Ado-Ekiti Southwestern, Nigeria. Inter Res J Agric Sci Soil Sci 3: 11-16.

7. Spironello A, Quaggio JA, Teixeira LAJ, Furlani PR, Sigrist JMM (2004) Pineapple yield and quality affected by NPK fertilization in a tropical soil. Rev Baraos Jabt 26: 155-159.

8. Sinclair E (1993) Leaf and soil analysis. Queensland fruit and vegetable growers. Pineapple field day. pp: 64-66.

9. Wondifraw T, Dawit A, Hailab A, Amsalu N, Tirfalem H (2006) Effects of stand 
Citation: Tewodros M, Mesfin S, Getachew W, Ashenafi A, Neim S (2018) Effect of Inorganic N and P Fertilizers on Fruit Yield and Yield Components of Pineapple (Annanas comosus MERR L. Var. Smooth cayanne) at Jimma, Southwest Ethiopia. Agrotechnology 7: 178. doi: 10.4172/21689881.1000178

Page 6 of 6

regulation on yield and quality of pineapple (Annanas comosus (L.) Merr. Var. Smooth cayenne), In Proceedings of the Inaugural and first Ethiopian horticultural science society conference, Addis Ababa, Ethiopia.

10. Ethiopian Institute of Agricultural Research (2007) Crop production technologies manual, Addis Ababa, Ethiopia. pp: 171-173.

11. FAO (2000) Guide lines for on farm plant nutrition and soil management trials and demonstrations. Rome, Italy.

12. Hermann D, Carole A, Kilovis F, Ndoumou D (2013) Impact of effective and indigenous microorganisms manures on Colocassia esculenta and enzymes activities. Afri J Agric Res 8: 1086-1092.

13. Tewodros M, Tadesse E, Getachew W, Mesfin S, Addisu B (2014) Pineapple production, postharvest utilization and marketing, production manual, Amharic version, EIAR.

14. IAR (1996) Progress and annual reports of Jimma agricultural research center, Jimma, Ethiopia.

15. Labouisse JP (2006) Summary of passport data of coffee germplasm maintained at Jimma Agricultural Research Center (JARC), Un-published.

16. Bremmer JM, Mulraney CS (1982) Nutrent Total In: Methods of soil analysis ( $2^{\text {nd }}$ edn) I: $565-624$.

17. Maclean EO (1982) Soil pH and lime requirement. In: methods of soil analysis part 2. Madison, USA, Amer Soc Agro 10: 199-234.

18. AOAC (2005) Association of Official Agricultural Chemist, Official Methods of Analysis $\left(17^{\text {th }}\right.$ edn) Vol. 1, 2. Horowitz edition intern, Maryland, USA

19. SAS Institute (2000) Statistical Analytical Systems SAS/STAT user's guide version $8(2)$ cary NC: SAS institute inc.
20. CIMMYT (1988) From Agronomic Data to Farmer Recommendations: An Economics Training Manual. Completely revised edition. Mexico, DF.

21. Babatola LA, Adebayo OB, Lawal OL (2002) Effects of different rates of poultry manure and NPK fertilizer on performance of Celocia argintia. Proceedings $16^{\text {th }}$ Annual conference of Horticultural Society of Nigeria, pp: 55.

22. Delali N (1995) Influence of nitrogen and potassium fertilizer ratio on yield and quality of pineapple (Ananas comosus MERR L Var. Smooth cayanne) in the southern forest-Savana ecotone of Ghana. MSc thesis presented at University of Ghana, pp: 110

23. Ademar S, Jose AQ, Teixelra LA, Sigrist JMM (2004) Pineapple yield and fruit quality affected by NPK fertilization in tropical Soil. 38: 263-274.

24. Abu T (2015) Effect of organic fertilizer water hyacinth on the growth and production of Taro [Colocasia esculenta (L.) Schott]. J Environm Eart Sci 5: 70-74.

25. Ayoola OT, Makinde EA (2007) Fertilizer treatments effects on performance of cassava under two planting patterns in a cassava-based cropping system in south west Nigeria. Res J Agric Biol Sci 3: 13-20.

26. Suminarti NE, Guritno B, Rayes ML (2016) Effect of fertilizer application and plant density on physiological aspect and yield of Taro [Colocasia esculenta (L.) Schott var. Antiquorum]. Inter J Agric Res 11: 32-39.

27. Orluchukwa J, Adedokun OM (2015) Response of growth and yield of pineapple (Ananas comosus) on Spent Mushroom substrates and inorganic fertilizer in South Nigeria. Inter J Plant Soil 8: 1-5.

28. Bussi C, Amicot MJ (1988) Effects of $N$ and $K$ fertilization on the growth, yield and pithum of apricot. J Horticul Sci Biotechnol 73: 387-392.

29. Bray RH, Kurtz LT (1955) Determination of total organic and available forms of phosphorus in soils. Soil Sci 39: 39-45. 